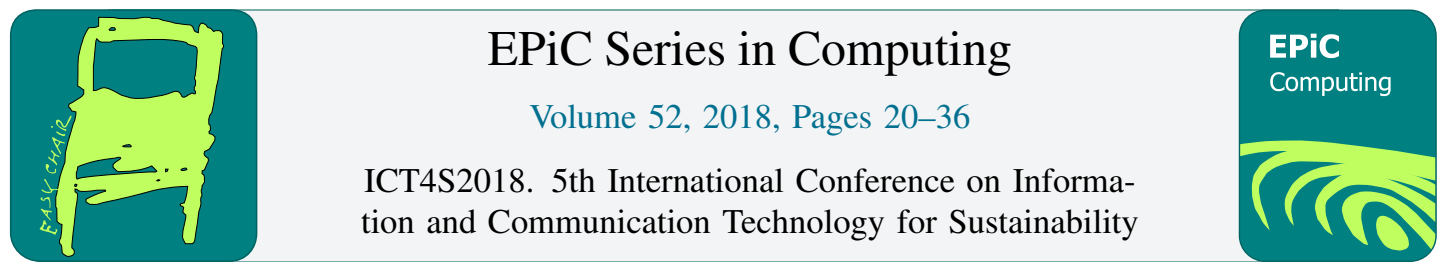

\title{
Evaluating Equality Requirements for Software Systems
}

\author{
Maryam Al Hinai ${ }^{1}$ and Ruzanna Chitchyan ${ }^{2}$ \\ ${ }^{1}$ Department of Informatics, University of Leicester, Leicester, UK \\ masahlele.ac.uk \\ ${ }^{2}$ Department of Computer Science, University of Bristol, Bristol, UK \\ r.chitchyan@bristol.ac.uk
}

\begin{abstract}
Equality is one of the key requisites of social sustainability: history shows that deprivation of some groups in favour of others inevitably leads to social tensions, unrest, and uprisings. As nowadays software systems control access to services, information, and even education, we maintain that all software systems ought to address equality requirements. To facilitate this, we present a template for equality requirements derived through study of 6 sample requirements specifications. The utility of the template is evaluated through a study of its application by a group of expert requirements engineers. The results obtained from a group that used the template are contrasted to the results from another group that completed the same task without the template. This study observes a substantial positive feedback from the template use.
\end{abstract}

\section{Introduction}

Equality is defined as the right for all members in a society to enjoy living and getting access to services and facilities without being discriminated because of their origin, believes, position, or (dis-)abilities [33]. History shows that deprivation of some groups in favour of others inevitably leads to social tensions, unrest, and uprisings [22]. This is well understood in most countries, where dedicated legislation is often put in place to guard for equality and fairness [15] that would help foster more socially sustainable societies.

As nowadays software systems control access to services, information, and even education, we maintain that all software systems too ought to address equality requirements. Furthermore, since equality is a notion applicable across all kinds of applications, independently on the application domains, it provides a good opportunity for equality requirements reuse.

Requirements reuse is often facilitated by requirements patterns [27] which improve the efficiency of the requirements engineering tasks [12]. Requirements patterns are models for logical grouping of requirements that can be adapted to fit different software systems and contexts [27]. These (relatively abstract) patterns are mapped onto detailed requirements [26] when instantiated within a specific context [12]. Often such patterns are represented through templates that ensure consistency $[27,26,31]$ and better reusability $[27,26]$ by helping to reduce efforts spent on discovering, organising and communicating requirements, and generating adequate requirements documentation $[27,30]$.

This paper presents a template for equality requirements (in section 2 ) which was previously detailed in [4]. The main focus of the paper is then on the evaluation of this template. The evaluation was undertaken through expert-based analysis with "think aloud" protocol. The set up of this study is presented in 
You can use any of the following templates to write the requirements statements.

- "[stakeholder] should [verb] [functions/services/resources] to accomplish [goal]".

E.g. [Legal authority] should [be able] to [post legal advice for home gardens] to [share legal best practices]

- "[stakeholder] with [variability] should [verb] [functions/services/resources] to accomplish [goal]".

E.g. [Citizen] with [different language] should [be allowed] to [select preferred language] to [customize the health inquiry application]

\begin{tabular}{|c|c|c|c|}
\hline \multirow{2}{*}{$\begin{array}{c}\text { Stakeholder } \\
(1)\end{array}$} & $\begin{array}{c}\text { Variability } \\
\text { (Stakeholders' gender, } \\
\text { age, language, religion, } \\
\text { position, income status, } \\
\text { knowledge, education, } \\
\text { race, technology used, } \\
\text { location etc.) } \\
(2)\end{array}$ & $\begin{array}{c}\text { Software } \\
\text { functions/services/resources } \\
\text { to achieve the goal } \\
(3)\end{array}$ & $\begin{array}{c}\text { Goal(s) } \\
(4)\end{array}$ \\
\hline e.g. Legal authority & - & $\begin{array}{c}\text { e.g. post legal advice for } \\
\text { home gardens }\end{array}$ & e.g. share legal best practices \\
\hline e.g. Citizen & e.g. Language & $\begin{array}{l}\text { e.g. Select preferred } \\
\text { language }\end{array}$ & $\begin{array}{l}\text { e.g. Customize application } \\
\text { language }\end{array}$ \\
\hline
\end{tabular}

Figure 1: Equality Template

section 3. Our study results are discussed in sections 4,5, 6. Threats to validity are discussed in section 7. Related work is summarised in section 8. The paper is concluded in section 9.

\section{Equality Requirements Template}

The pattern-based template development process generally commences with the input of knowledge sources (e.g., experts, past projects, standards documents, etc.) into the analysis process, whereby knowledge is synthesised into a pattern, a representation for the pattern is produced (e.g., as a template) which is suitable for reuse, after which the pattern/representation is continuously refined by collecting feedback from experts and users [26, 17, 35]. This is precisely the path that we took in eliciting the equality value pattern and its template-based representation (shown in Figure 1) [4].

To start with, using qualitative data analysis [23], we analysed a set of 11 papers on social sustainability (which specifically discussed equality). The study resulted in three main values related to equality. The values are equality with stakeholder variability, fairness in the selection of stakeholders goals, and equality with services and facilities. Equality with stakeholder variability value focuses on identification of factors that can differentiate stakeholders, e.g., according to their age, gender, literacy, etc (column 2 in Figure 1). Fairness is concerned with fair selection of stakeholder goals to be implemented in the system (column 4 in Figure 1), e.g., goals of stakeholders who have weaker influence may have large impact on social sustainability and so must be given thorough and fair consideration [4]. Equality for access to services is concerned with providing adequate services to all (diverse) types of stakeholders to enable them equal opportunities to complete a given goal (column 3 in Figure 1). For example, if a goal is to obtain information from a system, a visually impaired user should be able to use a software system same as a non-impaired user. In this case, large text fonts and customisable colour schemes or voice-based interfaces may be required. 


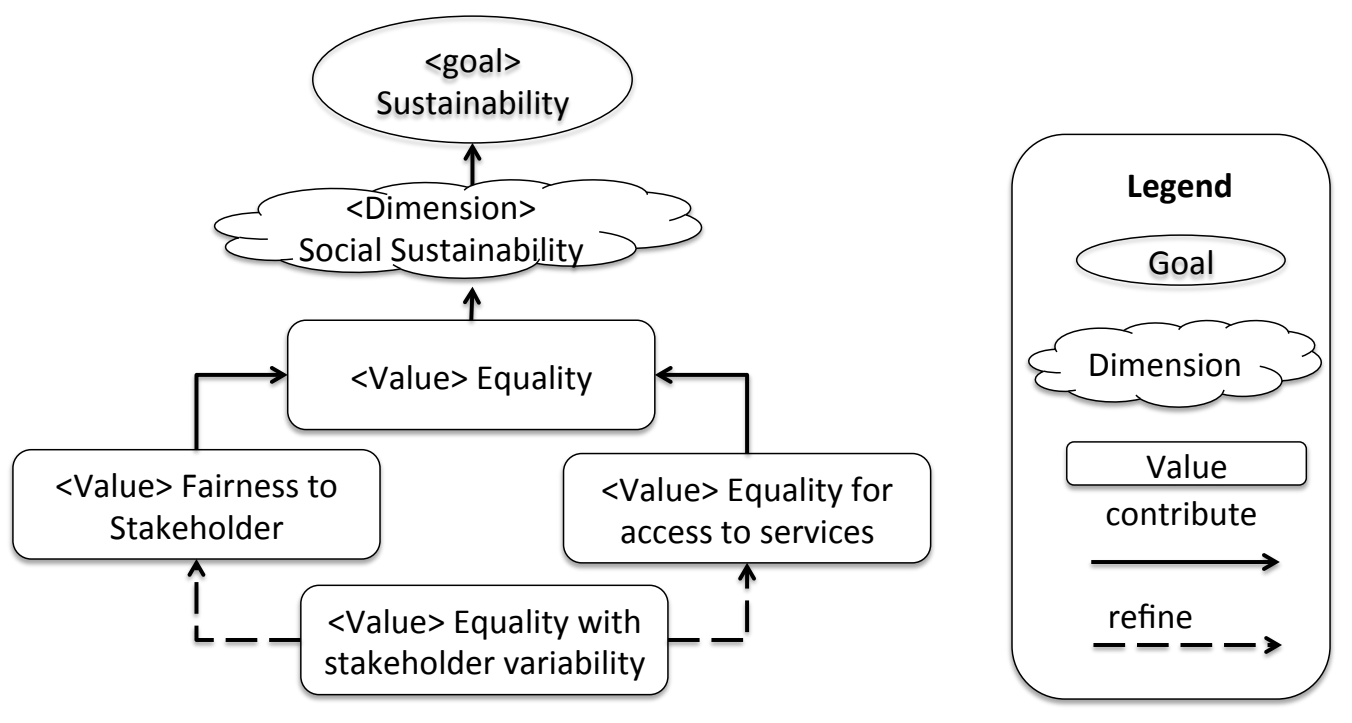

Figure 2: Equality value pattern

A set of questions were then suggested to operationalise the values into software requirements that support equality. This resulted in equality value pattern (shown in Figure 2) and equality requirements template (shown in Figure 1), where the template is designed to facilitate practical reuse of the equality value pattern. These pattern and template were then applied to 6 software requirements specification examples. More details on the template itself are provided in [4]. Below we report on an expert-based evaluation of the utility of our template.

\section{Study Design}

To evaluate the use and utility of the equality requirements template, we designed a study whereby two groups of experts undertook the equality requirements identification task for a given requirements brief. In one group, participants carried out the task without having equality template, while the other group was given the template and asked to use it during requirements elicitation. The study set off to address two research questions, namely:

$R Q 1:$ What do requirements engineers perceive as equality?

$R Q 2$ : Does the equality template facilitate the equality requirements elicitation?

The study subjects were requirements engineering experts and information systems analysts (who are also the intended users of the equality template). The template was expected to assist them with the identification of the equality requirements as well as with formulating these requirements for specification documents.

Eleven participants ( 5 female and 6 male) were recruited within the age range of 25 - 54 years old. Of these, 7 were academics and 4 industry practitioners. The participants self-specified their experience levels as 'expert' ( 1 practitioner), 'advanced' ( 4 academics and 1 practitioner), 'intermediate' ( 3 academics and 2 practitioners).

The participants were allocated into two groups, with as much skills and gender balance as possible (see Table 1). Those in Group 1 did the activity without using the equality template (for ease of identification in the rest of the article this group is referred to as NoTemp) while those in Group 2 used the 
Table 1: Study Participants

\begin{tabular}{|l|l|l|l|}
\hline Proficiency & Domain & $\begin{array}{l}\text { NoTemp } \\
\text { Group }\end{array}$ & $\begin{array}{l}\text { With- } \\
\text { Temp } \\
\text { Group }\end{array}$ \\
\hline Advance & Academic & 2 & 2 \\
\hline Intermediate & Academic & 1 & 2 \\
\hline Intermediate & Industrial & 1 & 1 \\
\hline Advance & Industrial & 1 & 0 \\
\hline Expert & Industrial & 0 & 1 \\
\hline \multicolumn{2}{|c|}{ Total } & 5 & 6 \\
\hline
\end{tabular}

equality template (further referred as WithTemp group). To ease readability the participants are referred to as NoTe-Px (for participant $\mathrm{x}$ in NoTemp group) or Te-Px (for participant $\mathrm{x}$ in with WithTemp group).

The task set before the study participants was to undertake equality requirements elicitation for a given requirements brief (see [2] for details on task formulation). The requirements brief pertained to a smart garden design [29] .

The participants were asked to use the think aloud protocol (also known as the verbal protocol analysis) [16]. With this protocol the study participants would continuously verbally comment aloud on the task they are handling, stating what they were doing, what are the reasons behind their decisions what are their opinions and why [16]. This protocol has been widely used for system design and evaluation [32], engineering design processes [5], usability testing [1,37, 36] and requirements analysis [28]. It was also well suited for our task: to explore whether requirements professionals found the equality template useful, as well as to see how use of the template would influence (either positively or negatively) the elicitation process.

All participants were given a copy of the information sheet, giving an overview of the activity and its reasons, as well as the materials for the actual task, which included: (i) a form to collect demographic information of the participants; (ii) instructions sheet detailing what exactly the participants should do (i.e., requirements elicitation for equality concern); and (iii) a stakeholders list. In addition, the WithTemp participants were furnished with the the equality value pattern and its requirements representation template, which they were asked to use for the elicitation activity.

As the participants started on the requirements elicitation task, their verbal commentary was recorded for further analysis (with their prior consent). A brief follow-up interview was conducted upon the task completion. (The full set of the activity materials is available from [2].)

The study design allowed for up to $30 \mathrm{~min}$. on the think aloud activity and up to $30 \mathrm{~min}$. for the follow-up interview. The actual time of each participant's engagement ranged between 30 to 40 minutes. The study was mostly conducted in English language. However, two participants wished to carry out the verbal commentary in Arabic - their mother tongue. This was acceptable, as it helped to remove communication barriers for the participants, and make them more comfortable with the activity [20]. Since the study lead is proficient in Arabic, she translated and transcribed the commentary upon completion.

The activity run as a relaxed interactive think aloud [37]: at times the researcher could minimise her intervention to acknowledgement tokens (e.g., 'yes', 'ok' ) in response to the participants' seeking confirmation (e.g., NoTe-P1: "You understand what I'm saying?" Researcher: "Yes."). On occasion, the researcher had to remind the participant to continue with verbalisation (e.g., telling to Te-P3 "Can you please keep talking"). Task continuation interventions [37] were used to encourage participants to continue identifying equality requirements (e.g., "What else do you think?" and "Anything to add?"). With some participants (Te-P6 and NoTe-P4), intervention was used to reduce their anxiety and to help 
Table 2: Category Codes

\begin{tabular}{|l|l|l|l|l|}
\hline \multirow{2}{*}{ Code } & \multicolumn{2}{|c|}{ NoTemp Group } & \multicolumn{2}{c|}{ WithTemp Group } \\
\cline { 2 - 5 } & No Part. & Refs. & No Part. & Refs. \\
\hline Accessibility & 4 & 17 & 5 & 29 \\
\hline Price affordability & 3 & 8 & 2 & 3 \\
\hline Inclusion & 2 & 16 & 4 & 9 \\
\hline Usability & 4 & 52 & 6 & 27 \\
\hline Functional Requirements & 3 & 29 & 6 & 23 \\
\hline Non-Functional Requirements & 4 & 24 & 6 & 43 \\
\hline Knowledge Management & - & - & 3 & 11 \\
\hline System Development & 4 & 19 & 6 & 21 \\
\hline
\end{tabular}

them through the activity $[10,9]$.

\subsection{Pilot study}

The study was piloted with 4 female software engineers, two of whom were 'advanced' postgraduates in Software Engineering and two were current intermediately-skilled in requirements $\mathrm{PhD}$ students. The pilot study was conducted to validate the structure of the think aloud protocol and the set task, in preparation for the full study.

The pilot study flagged a few necessary changes to the study materials' design, which were to:

1. Simplify the examples provided in the template;

2. Use colour coding in the template and the provided examples to make it simple for participants to follow and track the examples;

3. Include the value pattern diagram as an extra input material.

Upon consideration of the pilot results, the full evaluation study was conducted.

\subsection{Data Analysis}

The verbal protocol transcripts were analysed. The analysis was conducted using qualitative text analysis [23] technique, whereby key notions and concepts of interest from the transcript are identified, and categories into sets of related notions (labelled with a group code), and the possible relationships between the identified notions are studied and analysed. In this study we are interested in the relationship between the equality template use and the requirements elicitation process, specifically: Are the notions depicted in the template considered to be useful for the equality requirements identification? How does the template support/impede the elicitation process? Do the requirements identified with the template differ in any way from those identified without the template?

Thus, the text analysis was initiated with a set of pre-defined codes, where the codes represented the key notions supported via the template (i.e., stakeholders, variability, goals, services, fairness). Additionally, new codes were defined where respondents discussed notions not contained in the pre-defined code-set (e.g., system development, price affordability, inclusion, etc.). The code categories are listed in Table 2. The coding was conducted by the 1st author, verified by the 2 nd author and any differences were discussed and resolved, and results integrated. The analysis results are reported in section 4 below. 
Table 3: Stakeholders Elicited Per-Group

\begin{tabular}{|c|c|}
\hline NoTemp Group: No Template Used & With Temp Group: Equality Template Used. \\
\hline $\begin{array}{l}\text { - Users: people, users, team member, } \\
\text { owner (land, garden), consumers [gar- } \\
\text { den produce], society, supplier, "people } \\
\text { those who are from slum areas", "people } \\
\text { who are specially monitoring the plants, } \\
\text { monitoring the watering in our plants" } \\
\text { - Jobs: [seeds, plantation] analyst, gar- } \\
\text { dener,"somebody who will monitor ev- } \\
\text { eryday", public compound farmers, ex- } \\
\text { perts, developer, local support team } \\
\text { - Regulatory: department/ministry of } \\
\text { health, Government, } \\
\text { - Indirect stakeholders: society, nations } \\
\text { (e.g., people of Oman, UK or India) }\end{array}$ & $\begin{array}{l}\text { - Users: consumer, people living within } \\
\text { the area of the garden, customer, user, } \\
\text { producers, supplier, buyers } \\
\text { - Jobs: agriculturist, designers, landsca- } \\
\text { per, programmer, tester marketplace, } \\
\text { purchaser, owner of home garden, pri- } \\
\text { vate gardener hired by the owner, main- } \\
\text { tenance companies, housekeeping com- } \\
\text { panies, household, requirements analyst } \\
\text { gardener, consultant, team of opera- } \\
\text { tors, Maintenance and operations (also } \\
\text { referred to as: people for sowing and } \\
\text { maintaining the garden), procurement } \\
\text { department, help desk, environmentalist } \\
\text { - Regulatory: ministry of agriculture, citi- } \\
\text { zens, legal authority, regulator, sponsor, } \\
\text { ministry of environment, } \\
\text { - Hostile stakeholders: flies and insects } \\
\text { No of reference = } 132\end{array}$ \\
\hline
\end{tabular}

\section{Results and Discussion}

\subsection{Stakeholders}

Although both groups received the stakeholder list, participants with equality template were more specific in identifying the stakeholders (see Table 3). This was expected as the equality template instructs to relate stakeholders to various areas of equality requirements. This direct stakeholder identification and relation to requirements within the template is also the reason for the larger number of stakeholders having been identified by the participants from the WithTemp group. Here the group that used template reported 132 references to stakeholders vs 39 reported by the group that did not use the template.

Looking at the types of stakeholders identified, we observed that both groups discuss a number of consumer types, regulators and job roles. In addition, the group with template identified hostile stakeholder category, while the other group identified the category of indirect stakeholders.

\subsection{Variability}

As shown in Table 4, the WithTemp group identified more variability factors (84) than the NoTemp group (58).

Both groups have related equality requirements to education, age, language, disability and income. Although disability was not listed in the template, both groups used it. This might be due to the equality definition provided in the activity materials and due to its commonality in daily life issues. 
Table 4: Variability Elicited Per-Group

\begin{tabular}{|l|l|}
\hline $\begin{array}{l}\text { NoTemp Group: No } \\
\text { Template Used }\end{array}$ & $\begin{array}{l}\text { WithTemp Group: } \\
\text { Equality Template Used. }\end{array}$ \\
\hline $\begin{array}{l}\text { New or existing cus- } \\
\text { tomers, lifestyle, e.g. }\end{array}$ & $\begin{array}{l}\text { Gender, knowledge } \\
\text { (technological or gar- } \\
\text { 'don't have time to } \\
\text { dening), disability } \\
\text { read", garden features } \\
\text { (mental capacity), mem- } \\
\text { orisation capacity, race } \\
\text { ber of trees)* }\end{array}$ \\
\hline $\begin{array}{l}\text { Common: education, location, language, age, technology } \\
\text { used, position, disability (vision, deaf, blind), income status }\end{array}$ \\
\hline No of reference = 58 & No of reference = 84 \\
\hline *The participant mentioned the variability of the garden \\
features and emphasised that owners of any garden should be
\end{tabular}

The group with the template identified additional factors including race, culture, technology used, knowledge and memorisation capacity. Memorisation capacity was not listed in the template. This suggests that the template is flexible and can be modified for a specific case study.

One participant (NoTe-P2) from NoTemp group did not define any human variability factor and did not specify any inclusion, accessibility or usability requirements. This suggests that there is a possible relationship between variability value and identification of those requirements.

Participants in both groups thought of variability factors not only in terms of stakeholders but also in terms of the plant growth. These variability factors will vary based on the plants in the garden and this will affect the calculations and functions performed by the software. For example, NoTe-P2 identified that sunlight, plant age and fertilisers will affect the amount of water that will be calculated by the smart garden and Te-P2 identified soil type and the location of the garden.

Looking at the very large focus on verbalisation of variability factors (even in the NoTemp group), it is clear that equality is related to considering different/diverse people and allowing them to benefit from the system. This conforms to the equality value pattern suggested by this research.

Variability factors were also considered as barriers to the use of the system (NoTe-P1, "So education should not be a barrier"). In addition, Te-P6 looked at the variability as alternative interface design decisions (e.g., inputting date by typing vs. choosing a date from displayed calendar).

\subsection{Equality Requirements}

\subsubsection{Common Equality Requirements}

Both groups identified a number of common categories of equality requirements, including:

- Accessibility, e.g.: visual representation of information ("[the application should mention the quantity of water and the image depicting the level]" by NoTe-P5); easy navigation ("[access the history in a faster way. Also we can do it as a swipe]" by Te-P6), readability ("we have to make sure that the text is readable" by Te-P4).

- Affordable price, where the more affordable software is, the wider its' user segment (e.g., "providing [software] on affordable device at [an] affordable cost for common people [...] will promote equality" by NoTe-P3; "cheaper price so that the households could use this" by Te-P5). 
- Inclusion across geographic locations (e.g., "for instance, I am in Oman so the buttons should be in Arabic" by Te-P5) and languages (e.g., "we must have a different languages considered in the system" by Te-P4, and "We can change the language [to] Arabic or English by NoTe-P1).

- Accommodating differences between users (e.g.,"those who are not very good [with the] mouse can still use the system by just using the keyboard" by Te-P3, and "even when you provide list of values, you give pictures that will be more easy to use for any age group" by NoTe-P1), and allowing for the software to be used by marginalised groups of society (e.g., people [ ... ] who are from slum areas $[\ldots]$ can come on free basis and [ ... ] use this system") .

- Usability requirements identified by both groups relate to simplicity, error reduction and efficiency.

- Representative User Involvement into Software Development was noted as a key avenue which helps to ensure equality (e.g., Te-P5 suggested the use of an interaction design model since it is only by "involving the $[\ldots]$ intended users of these application[s]" where "the users are [...] the centre of the development" that equality would be assured. Similarly, NoTe-P3 stated that "you are going to be selecting the end user representation for agile rapid application or whatever it is. You have to go for some gender equality or some professional equality then the equality will appear in [the] software as well”. Similar directions of ensuring equality through design models and methods are discussed in related literature on participatory design techniques [8, 24].

Furthermore, group 2 identified a few group-specific requirements, as discussed below.

\subsubsection{Equality Requirements with Template}

In the WithTemp group two more requirement categories considered relevant to equality were identified:

- Availability was noted by Te-P1 (who suggested using hardware components that ensure application availability: "UPS because it has to work 24/7") and Te-P5 (who noted that the application needs to be available for use at any time: "I can just use the application [...] at any time and wherever I am").

- Knowledge Creation and Management category was noted by 3 participants (e.g., Te-P6 noted that equality will mean providing "[instruction on how to use the application]" and allowing gardeners to share their gardening experience through the software where "the users view each other['s] experience and experiments"; Te-P2 and Te-P3 suggested that consumers of garden products need to be aware of the smart garden system to know how the produce was grown.

\section{Participants' Viewpoint on Equality}

\subsection{Equality as Maximum Number of Users}

Participant NoTe-P1 viewed equality requirements as having the maximum possible number of users. This lead to identifying possible usage barriers and requirements that eliminate such barriers, so that the software application can reach the widest possible market (e.g., "anybody can buy and use it", "many people can use it"). NoTe-P1 considers as barriers what in this research is referred to as stakeholder variability factors (e.g., age, language, geographic location, etc ).

Furthermore, NoTe-P1 viewed supporting equality as addressing needs of humans in gradually larger social structures: from individuals, to communities, then regions and so on (e.g., "look at the individuals 
only but when you broaden it you will come to regions or countries..."). This also necessitated diverse development teams, e.g., "people are there [i.e., in a team] from different geographies", and "local support is needed" as local support teams contributes to equality by better representing local needs and requirements.

Participant NoTe-P5 views equality as allowing all types of gardeners to benefit from the application (e.g., "develop a system that can help them all") by reducing water consumption (e.g.,"allowing them to use the system based on either the number of trees or the area/size of garden"). The participant started by identifying different types of users and thinking about what each type would need to have in the application. She then sketched a user interface, helping herself to elicit requirements which would relate to interface decisions. Noting that the users "don't have time to read the information" and software use manuals, she suggested to represent information and interface as game icons: "like the Super Mario game, it shows the water, the water coloured in blue, trees in green in the application. [...] Consumption status indicator with colours (red, orange, yellow and green)". NoTe-P5 identified a new variability dimension (previously not considered in the template) - that is the lifestyle of the intended users (fast/busy).

Similarly, Te-P6 used the template for finding/anticipating problems that could be faced by users and defining requirements to solve them (e.g., "we can see that the language can be a barrier to using the system. The template gives us the functions that the system should have in addition to the goals".) Te-P6 thinks that the purpose of the template is: "to make it easier for the developer to know the requirements of an application. He can find [...] the problems currently faced by a user are and what the suggested solutions and goals are."

\subsection{Equality as Shared Responsibility for Software Project}

Participant Te-P1 defined equality as allowing all stakeholders to share the responsibility for making the software development project a success. This could be achieved by undertaking responsibilities and duties in the project: "So here, everyone has equal responsibility", "it is not one person who is running [the project]. All the stakeholders [...] mentioned here are part of this, equally involved in this".

Participant Te-P5 held the same view, with the slight difference that the users are to be considered the central players and engage into "interaction design".

Consequently, the template was primarily used to identify stakeholders who need to be part of the project, whether they represent individuals, departments or other systems.

\subsection{Equality as Indirect Stakeholder Support}

Participant NoTe-P3 considered indirect stakeholders to be the main focus of equality, so this category of stakeholders should not be neglected (e.g, "for each and every system certain social stakeholders or certain indirect stakeholders from [a] social aspect have to be added"). Negative stakeholders too must be given a consideration, else the "equality perspective will be in question". However, NoTe-P3 thinks that when software is developed for internal use in a specific company then, as a developer, one should not "... bother much about the equality of the society and all those factors". This is because such a system supposedly does not have either any societal impact or any indirect stakeholders. This, clearly, is an oversimplified view on equality, blinded to the diversity of the software system's stakeholders within the company (whether direct or indirect).

NoTe-P3 further relates equality to non-functional requirements such as affordability, and clarity (e.g., " clarity ... is fundamental requirement for equality. If clarity aspects [are] not there then inequality will come" because the software developers will interpret the requirements on their own, potentially neglecting equality/societal concerns). 


\subsection{System Functions vs. Equality}

Participant NoTe-P4 stated that there is no relationship between equality and the smart garden. This is because to her equality is narrowly defined as economic equality, which is supported by dedicated applications that monitor and report on economic differences between people. The other avenue through where software relates to equality, as per NoTe-P4, is when applications are specifically targeted at people with special needs and the deprived, e.g., for creating job opportunities (e.g., "if I am a disabled person ... and you give me the software that is really related to me giving me an opportunity to work"). However, for any other contexts, NoTe-P4 emphasised the difficulty of relating the concept of equality to software requirements. Only after viewing the template as part of the follow up interview, the participant started to grasp the possible connection between equality and software and suggested that delivery of "equal life knowledge to all the house owners" could be supported by providing video tutorials on planting and alike.

\subsection{System Functions and Stakeholder Selection}

Participant Te-P2 worked by using the template to gather what goals/tasks the current system is to complete. This system "as-it-is" [14] review is a usual starting point in requirements analysis, whereby the identified tasks shall be then transformed into the requirements statements for the software system. Te-P2 proceeded to consider:

- stakeholders as part of the system analysis team;

- variability factors as selection criteria for the representative stakeholders to be included in the analysis of the system (e.g.,"they have that right knowledge in the gardening . . . the knowledge, education and the technology use and a location should be indicated here . . . [as well as] the age.”).

\subsection{Equality as an Implied in Concern}

Participant Te-P3 viewed equality as part of accessibility and usability saying that "Normally we sum it up in one requirement like "user friendly" . . But when we talk of user friendly, inherently we are talking of equality because regardless of age, of gender or . . . the skill of the person" they should be able to use the system. Thus, equality is not an isolated requirement but "it's implied" as part of several other usability-related requirements. To this participant, the template served as a reminder: "Normally we tend to omit some . . . variability, but using your template it will keep on reminding us that this factor will also be important in the system that you are trying to produce".

Similarly, participant NoTe-P2 thinks of equality as an aspect embedded in Software Engineering principles, stating that equality is similar to the principles followed by engineers, where an engineer must consider the impact of his/her product "for society, for the customers, [...] with regards to, for example, privacy or patents or IP [ ... ] ", which are notions considered as part of the professionalism and ethics. The participant notes that although "equality is there already" within professionalism and ethics, "but to make it as one whole concept or one whole section of the requirement, this [is] my first time". To NoTe-P2 equality should be considered not only with respect to humans and "society as a whole" but also with respect to other creatures who may be stakeholders of the system as well. Thus, for the smart gardening system she notes the needs to consider the rights of the plants. 


\subsection{Equality as Stakeholders' Goal Support}

Participant Te-P4 used the template to identify stakeholders and analyse as to how the system could support their goals through software functions and features. She noted that variability can help refine a software service (e.g., in discussing how an application alters the user, she starts with smart phone users, then notes that "Some people use the cell phones only for texting and talking so maybe this person education or technology background doesn't help in using . . . software applications. So . . . system can send him a text massage if it's needed". She then observes that the age variability will require larger print fits for older people and blind users will need to be supported through voice messages.

\section{Discussion of Lessons Learned}

\subsection{Equality Requirements Identification Process}

By analysing the process that the two groups of requirements engineers used to identify equality requirements, we note that, irrespective of the template use, both groups looked at the variability factors and considered their affect on software functions and/or features, as well as at satisfying the stakeholders' goals through a software system. Thus, it is clear that stakeholders, variability, functions/features/services and goals are relevant contributors to the equality requirements and are commonly used by the requirements analysts.

At the same time, we observe that the equality elicitation process can start with any of the named factors and the sequence can vary depending on the analyst's perceptions, preferences, and convenience.

Where the analysts were asked to use the template, they tailored the template to their understanding of equality, e.g., by

- Using stakeholder variability characteristics as possible obstacles in reaching as many users as possible, and defining requirements to resolve these 'obstacles';

- Using the template in combination with stakeholder list (taken form the Onion model), whereby some stakeholders alternated between stakeholder selection and goal/function definition, where goals were defined and refined into function for each stakeholder (e.g., Te-P3, Te-P4) - filling in the template row by row. Some others participants started by identifying all possible stakeholders then reviewing all variabilities related to one stakeholder - i.e., aiming to fill the template in a more of a vertical manner (column by column fashion, as Te-P5);

- Goal and function were not used in all occasions (e.g., Te-P2), especially when the participants were working on the understanding the current functions of the intended software domain (i.e., gardening). At this point analysis is focused on stakeholders and what information they hold. More specific goals/functions for the software system would follow on form this. And in some cases goals come before while in other cases after the functions (e.g. Te-P4, Te-P6).

\subsection{Template Utility}

Overall, WithTemp group participants commented that the template was useful in identifying equality requirements. Participants commented that the template is simple (Te-P4, Te-P6), clear (Te-P3, TeP4), well organised (Te-P6), and sufficiently detailed (Te-P2). Participant Te-P6 noted that the template would be useful for system analysts to "extract" user requirements.

Participants Te-P3 and Te-P4 commented that the template serves as a good reminder on what should be considered by the analyst for equality: e.g., Te-P3 stated that "Normally we tend to omit some [ ... 
] variability, [ ... ] the template will keep on reminding us that this factor will also be important in the system ...", and Te-P4 said "It helped me a lot I was returning each time to it".

Several of participants from NoTemp group (who were not given the template during the experiment) had shown varying levels of misunderstanding about equality and its relation to software requirements. For instance, participant NoTe-P4 stated that there is no relationship between equality and the smart garden application because to her equality is being "treated as human beings". NoTe-P2 and NoTe-P1 thought that equality is often implied as part of other requirements (e.g., NoTe-P1 stated: "when you say portability, equality may not come in the first phase but when you dig [deeper], equality is there"). NoTe-P2 further noted that the present study was the first time that she had seen the notion of equality as "whole concept or one whole section of the requirement". This indicates that software developers may omit equality concerns because they do not see the immediate link between equality and software requirements. They may also part-specify equality as it relates to other concerns, but fail to consider the notion as a whole.

All this demonstrates the three key areas of the present template utility:

- The template tackles the issue of conceptual misunderstanding of equality by depicting a direct and clear association between the equality concept and software requirements;

- The template consolidates the notion of equality into a coherent structure, rather than leaving it as implied across several other concerns;

- The template acts as a visual reminder on the variability dimensions that should be considered for all stakeholder types to assure equality.

\subsection{Other Avenues for Equality Support}

During the follow up interview, the participants were asked how would they suggest to improve equality consideration in software engineering. Some of the provided suggestions are outlined below:

- Equality metrics should be used from the early stages of development, these metrics should ideally be "already embedded in the methodology" (NoTe-P3). This is because the metrics that require measurement and monitoring without integration into the originally used methodology will impose other tasks and will not be well received by the developers. This is in line with related work on social sustainability, e.g., Groher and Weinreich reported that the lack of such measuring tools contributes to sustainability challenges in software development [18].

- Equality standards could be defined by the professional bodies in SE "like, for example, when we have the principle with the ACM standard so we can have that as guideline" (NoTe-P2).

- Equality concerns could be taught as part of the software engineering curriculum, ensuring that the students (who are future practitioners) are familiar with the equality concerns and solutions, thus integrating these into their own future developed products/processes (NoTe-P2).

- Equality criteria checklists can be created to validate that the equality concern is adequately handled in the given requirements (NoTe-P4).

- Governments could legislate the necessity to consider equality in all software products (NoTe-P2 and NoTe-P4).

We observe that all the provided suggestions are complementary to the template use, and could be integrated with the template use process. 


\section{Threats to Validity and Limitations}

\subsection{External Validity}

A possible threat to external validity is related to the sample size which can affect the generalisability of findings. However, using small-sized samples is a common practice in think aloud protocol-based studies [28]. In a well-cited paper J. Nielsen [25] estimated the sufficient number of participants in a think aloud activity to be $4 \pm 1$. Reporting on think-aloud activity with a similarly-sized sample is, thus, not unusual (e.g., [13] with 5 participants in each group). Nevertheless, additional studies would be necessary to confirm the generalisability of the present findings.

Another threat arises from the allocation of participants into groups. Although the groups were formed with care for a balanced representation of gender, experience and background, we used participants' reported experience rather than experimentally validated level of individual expertise. Consequently, it is possible that more skilled (in the related questions) participants, happened to be grouped together in one of the groups. Nevertheless, a great deal of various positive feedback from the participants about the template clearly demonstrates its importance and effectiveness.

Related to the previous point is also the participants' prior familiarity with the think aloud protocol. The study did not require that the participants should have prior knowledge of this technique, though the information sheet, sent to participants before the study task, explained that a think aloud protocol would be used. Out of all the participants only one (Te-P5) commented on prior familiarity with such protocols. However, if the lack of familiarity with the protocol were to be a compounding issue, both groups would be similarly affected. Furthermore, the simplicity of the protocol itself helps with ameliorating this treat.

Another possible threat arises from the software case provided (i.e. smart garden) and from the context of the study. The selected case relates to a smart garden support software system. This system would, from the first sight, have nothing to do with equality. While it is true that other (e.g., more socially-focused) systems would bring up more equality concerns, we intentionally chose a non-obvious system for social concerns, as we maintain that the equality notions manifest (possibly to a varying degree) in all software systems. This, indeed, is confirmed by the study results, as discussed above.

The brief given to the participants was a simplification of a real-world application (to allow activity completion within the available study time). While, in reality, requirements engineers would be able to get input from the system stakeholders, for the purposes of this study they were advised to use assumptions to cover any gaps and missing information. As a result, the views from system stakeholders are missing in this study. Yet, as the intend of this study was to focus on the perceptions and practices of software professionals, we consider this simplification to be appropriate for the intended purpose.

Finally, a possible threat to result generalisability comes from the sample selection procedure: the participants were reqruited from the authors' personal contact cycles. We endeavoured to mitigate this treat by selecting people from various backgrounds and from various countries. Furthermore, though the participants were known to us, we had no personal relationships or conflicts of interests with them.

\subsection{Conclusion Validity}

Given the qualitative nature of this work, there are no conclusions related to the statistical validity. Yet, to assure consistent measurements during the study implementation, the activities were standardised across the individuals and groups as much as possible: all participants were provided with identical materials (as relevant per group activity) and minimum timings were observed. In addition, a follow-up semi-structured interview was conducted following a pre-defined interview guide. 


\subsection{Internal Validity}

A major concern to the presented results is the treat that the prior understanding of equality by the participants could be a confounding factor to the way that template was used. In an ideal world, the researchers would be able to establish a clear understanding of equality (e.g., an ontology), train the template users in this "understanding", then evaluate the template use in accordance with it. However, this kind of experiment design would rather defeat the point of the present work. Unlike the idealised normative worldview, we take a descriptive standpoint: we expect that there are substantial differences in the way that equality is perceived in communities and between individuals ${ }^{1}$. These differences could have a substantial bearing on the utility of the proposed pattern and template. We are interested in finding out if, despite any differences, the template is/ is not perceived to be useful. An average intended user of this template would not be expected to have a "standartised" perception on what equality should be exactly; and we intend for it to be possible to use this template within any given requirements elicitation and specification process. Thus, the present study looks at the questions of equality perception and template use together. Though a brief definition of equality is provided to all study participants, it is non restrictive. In short, in this paper, we do not isolate the pre-existing notions of equality as a confounding factor, but study it along (under RQ1) with the template use (under RQ2).

Finally, by conducting a pilot test as (described in Section 3.1), a possible threat related to the instrumentation (i.e., the materials used in the activity) was mitigated.

\section{Related Work on Equality}

A comparative study on assessment of the social impact of building materials and identification of the most socially sustainable ones is presented in [19]. This study also aimed at identifying trade-offs and potential improvements in stages of the building process. The indicators of social impact considered here include fair salary, equal opportunities and discrimination in different building phases (pre-building, building and post-building phase). In addition, fair competition between value chain actors was used as an indicator for social impacts on value chain members [19].

In [7], the long-term social performance of irrigation was assessed using a compound social viability indicator. This viability indicator was subcategorised to social impact and social capacity. According to [7], "social impact refers to the effects of irrigation on people, their well-being, social organisation, and livelihoods." The paper notes that equal water distribution between users can be an indicator to equality [7]. Yet, in some cases equality is differentiated from fairness and fair distribution (not equality) is used as social sustainability indicator [7].

In [11], Chitb and Komathi suggest a model for evaluating vulnerabilities of the development of ICT programs in rural areas. Socio-cultural vulnerabilities are linked to the relationships among community members [11]. The human relationships "affect access to resources and assets, and decision-making power of people, established by gender, age, race, religion, caste, and class egalitarianism within communities" [11]. The paper suggests that ICT interventions help in reconciling the vulnerabilities by reducing the gender, social and economic power [11].

Gender equality was also an indicator of social sustainability in the study where a framework for sustainable development policy was suggested [34]. The paper argued that reducing the gap between genders satisfies love and belonging needs of the members of a community [34]. Economic participation and opportunity, educational attainment, political empowerment and health and survival were sub-indicators of gender gap (attained from Gender Gap Index GGI) [34]. "The GGI provides a measure of societal/jurisdictional views toward gender equality" [34].

\footnotetext{
${ }^{1}$ Study of nuanced differences in equality perception within cultures and communities is beyond the scope of this work, and belongs to the area of Humanities.
} 
The study in [38] was conducted with a goal of achieving sustainable development that balances the economic, environmental and social aspects of sustainability. One of selected social sustainability indicators was equity in income level between rural and urban areas/residents [38].

Social equity was selected as a dimension of social sustainability evaluation suggested in [21]. Social equity "measures the level of equality in the way resources and opportunities are distributed in a community" [21]. The resources and opportunities included housing, community (e.g. child care) and social (e.g. cultural events) infrastructure [21].

The indicators of income equality, gender equality, equal resources and services distribution, collected from the papers included in the literature review of [3] are promoted as social sustainability characteristics. However, not all of these indicators are directly quantifiable or measurable: e.g., it is not clear how to undertake the measurement of monopoly and anticompetitive behaviours to evaluate fair competition indicator between value chain actors. Yet, all these indicators are, without a doubt, relevant and important concerns that must be taken into consideration during software engineering if the intended systems are to promote equality. We observe that these indicators often correspond to more detailed breakdown of equality-related values held in the given communities.

Barn states in [6] that "ownership and property; privacy, freedom from bias, universal usability, trust, autonomy, informed consent, identity and others" are the most applicable values to Information systems. We can see that these are rather similar to equality/social sustainability indicators.

\section{Conclusion}

In this paper, we presented an evaluation of our equality requirements elicitation template. The evaluation was undertaken by experts who participated in a think aloud activity. We set out to investigate two research questions. One is What do requirements engineers perceive as equality? and the second is Does the equality template facilitate the equality requirements elicitation?

For the first question, the results in 4.3 show that experts perceive equality as accessibility, price affordability, inclusion and accommodation of users with differences, usability, user involvement and representation in the software development process. However, the notion of social justice seem to be underrepresented in these perceptions. Using the template in equality requirements elicitation process ensures that there is just distribution in terms of goals and access as well.

For the second question, the template helped to bring the otherwise distributed and implicit notion of equality into explicit view, it served as a reminder to address various variability notions, which otherwise could be omitted/forgotten. And, most importantly, the template tackled the conceptual misunderstanding that equality is not relevant to some software applications.

\section{References}

[1] S. A. Abdulhak, K. H. Cha, and D. K. Kang. Evaluation of online social network games usability using verbal protocol analysis. In 2011 6th International Conference on Computer Sciences and Convergence Information Technology (ICCIT), pages 7-11, November 2011.

[2] Maryam Al Hinai. Instruments for a think aloud study. Available at https://drive.google.com/ open? id=1hXX497H_RqwH6sQc0JoCtkGj17KhOrh6.

[3] Maryam Al Hinai and Ruzanna Chitchyan. Social sustainability indicators for software: Initial review. In Birgit Penzenstadler, Martin Mahaux, and Camille Salinesi, editors, Proceedings of the 3rd International Workshop on Requirements Engineering for Sustainable Systems (RE4SuSy'14), pages 21-27, Karlskrona, Sweden, Aug 2014.

[4] Maryam Al Hinai and Ruzanna Chitchyan. Engineering requirements for social sustainability. In ICT for Sustainability 2016. Atlantis Press, 2016. 
[5] Cynthia J Atman, Justin R Chimka, Karen M Bursic, and Heather L Nachtmann. A comparison of freshman and senior engineering design processes. Design Studies, 20(2):131 - 152, 1999.

[6] Balbir Barn, Ravinder Barn, and Franco Raimondi. On the role of value sensitive concerns in software engineering practice. In Proceedings of the 37th International Conference on Software Engineering - Volume 2, ICSE '15, pages 497-500, Piscataway, NJ, USA, 2015. IEEE Press.

[7] M.G. Bos, D.H. Murray-Rust, D.J. Merrey, H.G. Johnson, and W.B. Snellen. Methodologies for assessing performance of irrigation and drainage management. Irrigation and Drainage Systems, 7(4):231-261, 1993.

[8] Sandra Buchmüller, Gesche Joost, Nina Bessing, and Stephanie Stein. Bridging the gender and generation gap by ict applying a participatory design process. Personal and Ubiquitous Computing, 15(7):743-758, October 2011.

[9] Jacob Buur and Kirsten Bagger. Replacing usability testing with user dialogue. Commun. ACM, 42(5):63-66, May 1999.

[10] Phil Carter. Liberating usability testing. interactions, 14(2):18-22, March 2007.

[11] Arul Chib and A.L.E. Komathi. Extending the technology-community-management model to disaster recovery: Assessing vulnerability in rural asia. In Information and Communication Technologies and Development (ICTD), 2009 International Conference on, pages 328-336, April 2009.

[12] Robert Darimont, Wei Zhao, Christophe Ponsard, and Arnaud Michot. Deploying a template and pattern library for improved reuse of requirements across projects. In 25th IEEE International Requirements Engineering Conference, RE, page 2, 2017.

[13] Abraham Nieva de la Hidalga, María Auxilio Medina Nieto, María Leticia Ramírez Castillo, Antonio Benitez Ruiz, and Jorge de la Calleja Mora. Adaptation of biovel portal: a usability case study. In Software Engineering Research and Innovation (CONISOFT), 2016 4th International Conference in, pages 185-194. IEEE, 2016.

[14] Alan Dennis. Systems Analysis and Design. John Wiley \& Sons, Inc., 5th edition, 2012.

[15] Equality Act 2010 c.15, 2010. Accessed: 04-08-2017.

[16] K Anders Ericsson and Herbert A Simon. Verbal reports as data. Psychological review, 87(3):215, 1980.

[17] Erich Gamma, Richard Helm, Ralph Johnson, and John Vlissides. Design patterns: elements of reusable object-oriented software. Addison-Wesley Professional, 1994.

[18] Iris Groher and Rainer Weinreich. An interview study on sustainability concerns in software development projects. In Software Engineering and Advanced Applications (SEAA), 2017 43rd Euromicro Conference on, pages 350-358. IEEE, 2017.

[19] Seyed Abbas Hosseinijou, Saeed Mansour, and Mohsen Akbarpour Shirazi. Social life cycle assessment for material selection: a case study of building materials. The International Journal of Life Cycle Assessment, 19(3):620-645, 2013.

[20] Elekes Katalin. "please keep talking": The 'think-aloud' method in second language reading research. Novelty, 7(3):48-59, 2000.

[21] Chris Landorf. Evaluating social sustainability in historic urban environments. International Journal of Heritage Studies, 17(5):463-477, 2011.

[22] Bo Malmberg, Eva Andersson, and John Östh. Segregation and urban unrest in sweden. Urban Geography, 34(7):1031-1046, 2013.

[23] Matthew B. Miles, A. Michael Huberman, and Johnny Saldaña. Qualitative data analysis: A methods sourcebook. SAGE, 3 edition, 2014.

[24] Usman Mushtaq and Kevin Hall. Power and agency in health information technology: towards a more meaningful participatory design for sustainable development. In 2009 IEEE Toronto International Conference Science and Technology for Humanity (TIC-STH), pages 1036-1041, September 2009.

[25] Jakob Nielsen. Estimating the number of subjects needed for a thinking aloud test. International journal of human-computer studies, 41(3):385-397, 1994.

[26] Maria Riaz and Laurie Williams. Security requirements patterns: understanding the science behind the art of pattern writing. In Second IEEE International Workshop on Requirements Patterns, RePa 2012, Chicago, IL, 
USA, September 24, 2012, pages 29-34, 2012.

[27] Suzanne Robertson and James Robertson. Mastering the Requirements Process: Getting Requirements Right. Addison-Wesley, 3 edition, 2013.

[28] K. D. Schenk, Nicholas P. Vitalari, and K. Shannon Davis. Differences between novice and expert systems analysts: What do we know and what do we do? Journal of Management Information Systems, 15(1):9-50, 1998.

[29] Resilient smart gardens. Accessed: October 2016.

[30] W Spencer Smith and Lei Lai. A new requirements template for scientific computing. In Proceedings of the First International Workshop on Situational Requirements Engineering Processes-Methods, Techniques and Tools to Support Situation-Specific Requirements Engineering Processes, SREP, volume 5, pages 107-121. Citeseer, 2005.

[31] Ian Sommerville. Software Engineering, Global Edition. Pearson Education Limited, 10 edition, 2016. Accessed: 27-09-2017.

[32] Neville A. Stanton, Paul M. Salmon, and Guy H. Walker. Human Factors Methods: A Practical Guide for Engineering and Design. Ashgate Publishing Ltd, 2007.

[33] Understanding equality. Accessed: 06-08-2017.

[34] Philip R. Walsh. Creating a "values" chain for sustainable development in developing nations: where maslow meets porter. Environment, Development and Sustainability, 13(4):789-805, 2011.

[35] Stephen Withall. Software Requirement Patterns. Pearson Education, 2007.

[36] Po-Yin Yen and Suzanne Bakken. A comparison of usability evaluation methods: heuristic evaluation versus end-user think-aloud protocol-an example from a web-based communication tool for nurse scheduling. In AMIA, 2009.

[37] Tingting Zhao and Sharon McDonald. Keep talking: An analysis of participant utterances gathered using two concurrent think-aloud methods. In Proceedings of the 6th Nordic Conference on Human-Computer Interaction: Extending Boundaries, NordiCHI '10, pages 581-590, New York, NY, USA, 2010. ACM.

[38] Lin Zhen, Shuyan Cao, Yunjie Wei, Oliver Dilly, Xuelin Liu, Fen Li, Hannes Koenig, Karen Tscherning, and Katharina Helming. Comparison of sustainability issues in two sensitive areas of china. Environmental Science and Policy, 12(8):1153 - 1167, 2009. 